\title{
Unloading RNAs in the cytoplasm
}

\section{An "importin" task}

Sandra M.G. Dias, ${ }^{1}$ Richard A. Cerione ${ }^{1,2, *}$ and Kristin F. Wilson ${ }^{1}$

${ }^{1}$ Department of Molecular Medicine; College of Veterinary Medicine; ${ }^{2}$ Department of Chemistry and Chemical Biology; Baker Laboratory; Cornell University;

Ithaca, NY USA

Key words: cap binding protein, capped mRNA, capped U snRNA, importin- $\alpha$, importin- $\beta$, $\mathrm{CBC}$ regulation

Abbreviations: mRNA, messenger RNA; snRNA, U small nuclear RNA; CBC, nuclear cap-binding complex; eIF-4E, eucaryotic initiation factor- $4 \mathrm{E}$

Submitted: 11/20/09

Accepted: 12/02/09

Previously published online: www.landesbioscience.com/journals/ nucleus/article/10919

*Correspondence to: Richard A. Cerione; Email: rac1@cornell.edu

Commentary to: Dias SM, Wilson KF, Rojas KS, Ambrosio AL, Cerione RA. The molecular basis for the regulation of the cap-binding complex by the importins. Nat Struct Mol Biol 2009; 16:930-7; PMID: 19668212; DOI: 10.1038/nsmb.1649.
$\mathrm{T}$ he nuclear cap-binding complex (CBC), a heterodimer comprised of a $20 \mathrm{kDa}$ subunit (CBP20) and an $80 \mathrm{kDa}$ regulatory subunit (CBP80), binds to nascent RNA polymerase II transcripts and is important throughout different aspects of RNA metabolism. In a recent publication, using a combination of X-ray crystallographic information, mutagenesis studies, smallangle scattering experiments, analytical ultracentrifugation and in vivo assays, we presented evidence that importin- $\alpha$ and importin- $\beta$, two nucleocytoplasmic transport proteins, play key roles in regulating the binding of capped RNA by the CBC in cells. A model for how complexes between $\mathrm{CBC}$ and the importins cycle in and out of the nucleus and direct the proper positional binding and release of capped RNA is presented here and is discussed in light of recent publications.

\section{Regulation of the Nuclear and Cytosolic Cap-Binding Proteins}

In eukaryotes, mRNAs and U snRNAs are modified as a result of the addition of a 7-methyl guanosine cap $\left(\mathrm{m}^{7} \mathrm{G}\left(5^{\prime}\right)\right.$ $\left.\operatorname{ppp}\left(5^{\prime}\right) \mathrm{N}\right)$ to its $5^{\prime}$-end, which is essential for the recognition and binding of two RNA-binding proteins, the cytosolic protein eIF-4E and the nuclear cap-binding complex (CBC). eIF-4E along with eIF-4G and eIF-4A forms the eIF-4F complex, which is crucial for recruiting the small ribosomal subunit to the mRNA 5 '-end and for subsequent searching for the start codon and protein translation (reviewed by von der Haar et al.). ${ }^{1}$
The CBC is comprised of a $20 \mathrm{kDa}$ capbinding subunit, CBP20, and an $80 \mathrm{kDa}$ subunit, CBP80, that enables CBP20 to bind the cap with high affinity. This complex is involved in a number of different aspects of RNA processing, including the regulation of the splicing efficiency of cap-proximal introns, the stimulation of 3 '-end formation, the inhibition of mRNA de-adenylation, the export of $U$ snRNAs and mRNAs (reviewed in refs. 2 and 3), as well as the mediation of a pioneer round of translation for mRNAs subject to nonsense-mediated decay ${ }^{4,5}$ (reviewed in ref. 6).

It is well established that the binding of capped RNA by eIF-4E is under strict growth factor regulation. In particular, growth factor-dependent signaling through mTOR has been shown to mediate the phosphorylation of a family of proteins, designated the eIF-4E-binding proteins (4E-BPs), that negatively regulate the cap-binding activity of eIF-4E (reviewed in ref. 1 and 7). Growth factordependent phosphorylation of the 4E-BPs triggers their release from eIF-4E, enabling eIF-4E to bind to eIF-4G, thereby significantly increasing its affinity for cap. Thus, growth factor-dependent signaling enables the cytosolic cap-binding protein eIF-4E to switch between a negative regulator $(4 \mathrm{E}-\mathrm{BP})$ and a positive regulator (eIF-4G) and in this way control when the binding and translation of capped mRNA can begin. Likewise, we have found that the other major cap-binding complex in cells, the CBC, is under tight growth factor regulation. ${ }^{89}$ This growth factor signaling pathway also involves mTOR and 
p70 S6K and culminates in the increased cap-binding capability of the CBC. However, the molecular basis for this regulation has until now been unclear.

\section{"Importin" Regulators}

Importin- $\alpha$ binds to the nuclear localization sequence (NLS) within the $\mathrm{N}$-terminal end of the CBP80 subunit and participates together with importin- $\beta$ in the nuclear import of the CBC. ${ }^{10-13}$ However, in addition to their classical roles in nucleocytoplasmic transport, the interactions between the importins and the $\mathrm{CBC}$ have also been shown to exert an influence on cap-binding activity in vitro. ${ }^{11}$ While the CBC-importin- $\alpha$ complex is able to bind capped RNA with high affinity, the inclusion of importin- $\beta$ into this protein complex stimulates the release of capped RNA. In vivo, the inter-conversion between the CBC-importin- $\alpha$ and CBC-importin- $\alpha$-importin- $\beta$ complexes would be regulated by Ran-GTP (i.e., the binding of Ran-GTP to importin- $\beta$ causes it to dissociate from the CBC-importin- $\alpha$ complex). Therefore, because Ran-GTP is a nuclear species, it has been suggested that the CBC-importin- $\alpha$ complex would bind to capped RNA in the nucleus, whereas the formation of the CBC-importin- $\alpha$ importin- $\beta$ complex within the cytosol would then result in the directional release of capped RNA.

The possibility that the importins could function to "unload" capped RNA in the cytosol is intriguing, especially given the recent work by Worch R, et al. ${ }^{14}$ which suggests that the $\mathrm{CBC}$ has a much higher affinity for capped RNA than does eIF4E. In short, by using fluorescence and surface plasmon resonance, they compared the kinetics of the $\mathrm{m}^{7} \mathrm{GpppG}$-eIF-4E $\mathrm{E}^{15,16}$ and $\mathrm{m}^{7} \mathrm{GpppG}-\mathrm{CBC}$ interactions, and found that the association of capped RNA transcripts with $\mathrm{CBC}$ was strongly favored from both thermodynamic (equilibrium) and dynamic (kinetic) considerations. Additionally, the cellular concentration of eIF-4E was estimated to be very low $\left(\sim 8 \times 10^{5}\right.$ molecules per HeLa cell) ${ }^{17}$ compared to the CBC $\left(\sim 7 \times 10^{9}\right.$ molecules per HeLa cell). ${ }^{18}$ Taken together, these data would suggest that the $\mathrm{CBC}$ would remain bound to capped-RNA in the presence of
eIF-4E unless the RNA was actively displaced from the CBC.

We also had observed an extremely stable interaction between the $\mathrm{CBC}$ and importin- $\alpha$ both in vitro and in vivo (Wilson K, unpublished data). The fact that the importins had been shown to regulate cap-binding in vitro, ${ }^{11}$ caused us to question whether differential complex formation between the $\mathrm{CBC}$ and the importins might underlie the molecular basis for the growth factor-induced cap-binding changes which we had observed. ${ }^{8,9}$ As a first step, we confirmed that the CBC could bind to capped-RNA with high affinity in the presence of importin- $\alpha$, but would not bind the capped-RNA upon addition of importin- $\beta$. The next step was to understand how the importins conferred these regulatory effects onto the $\mathrm{CBC}$.

\section{The Crystal Structure of the CBC-Importin- $\alpha$ Complex Provides "Importin" Clues}

To this end, we started by solving the $\mathrm{X}$-ray crystal structure of the $\mathrm{CBC}$ bound to importin- $\alpha \Delta$ IBB (an importin- $\alpha$ construct in which the importin- $\beta$ binding domain had been deleted) to a 2.2 $\AA$ resolution. This structure revealed that many of the features exhibited by the three proteins within the complex (i.e., CBP20, CBP80 and importin$\alpha \Delta \mathrm{IBB})$ were similar to those described previously from X-ray crystal structures of the independent components. ${ }^{19,20}$ The interaction between the first 21 residues of CBP80 containing the NLS and importin$\alpha \triangle \mathrm{IBB}$ covered a solvent-accessible area within importin- $\alpha \Delta \mathrm{IBB}$ of $1,175 \AA^{2}$, as calculated by PISA. ${ }^{21}$ Within this region there were 34 hydrogen bonds and 12 salt bridges, as well as a number of hydrophobic contacts between the side chains from CBP80 and conserved tryptophan residues from importin- $\alpha \Delta$ IBB. These abundant interactions likely explain why the inclusion of importin- $\alpha$ within the cap-binding complex, which has been documented through multiple stages of RNA processing, ${ }^{13}$ is well conserved from protozoa through eukaryotes. ${ }^{11-13}$

One surprising finding, however, was that despite the fact that $\mathrm{m}^{7} \mathrm{GpppG}$ was co-purified with the complex, the cap analogue was absent from the structure. In the place of $\mathrm{m}^{7} \mathrm{GpppG}$, there was an importin- $\alpha$ molecule from a symmetry partner making key contacts with residues from CBP20 normally involved in cap binding. It was this unexpected result that prompted us to consider that importin- $\beta$, in a similar fashion, might be playing this role within the context of the CBCimportin- $\alpha$-importin- $\beta$ tetramer as a means for triggering the release of capped RNA from the CBC in the cytoplasm.

\section{Discerning the Importin- $\beta$ Trigger}

To explore this question, we wanted to test the somewhat counter-intuitive idea that importin- $\beta$ could bind the $\mathrm{CBC}$, and in particular CBP20, directly. We hypothesized that if importin- $\beta$ could displace capped RNA, it might do so by binding to regions on CBP20 typically involved in cap-binding. Thus, we started by looking at an interaction between CBP20 and CBP80 that was speculated to help CBP80 confer high affinity cap-binding on CBP20. ${ }^{19}$ Mutating residues Lys327 and Glu328 on CBP80 (residues in pink-inset Fig. 1), which form a "binding groove" that interacts with the first 14 residues from CBP20 (i.e., helices $\alpha 1-\alpha 2$, Fig. 1, inset, yellow), weakened the CBCcap interaction in a fashion analogous to the deletion of the first 14 residues of CBP20. While CBP80 does not contact the cap structure directly, ${ }^{19}$ it appears to have an important stabilizing effect on the $\alpha 2-\alpha 3$ loop of CBP20 (Fig. 1 and inset, red) and positively affects the $\pi-\pi$ stacking interactions between Tyr 20 and Tyr 43 of CBP20, and the methyl guanosine ring of the cap structure (Fig. 1 inset, residues in sticks red, cap in sticks yellow). These data suggested that one way in which importin- $\beta$ might function to stimulate the release of capped RNA would be to compete with CBP80 for binding of the first 14 amino acids on CBP20.

We also demonstrated that the deletion of the last 30 residues on CBP20 (i.e., residues 126-156 which include Tyr138) affected the cap-binding capability of the $\mathrm{CBC}$, thereby pointing to another region on CBP2 20 where contact with importin- $\beta$ could have a functional effect. The importance of Tyr138, as well as Tyr20 and 


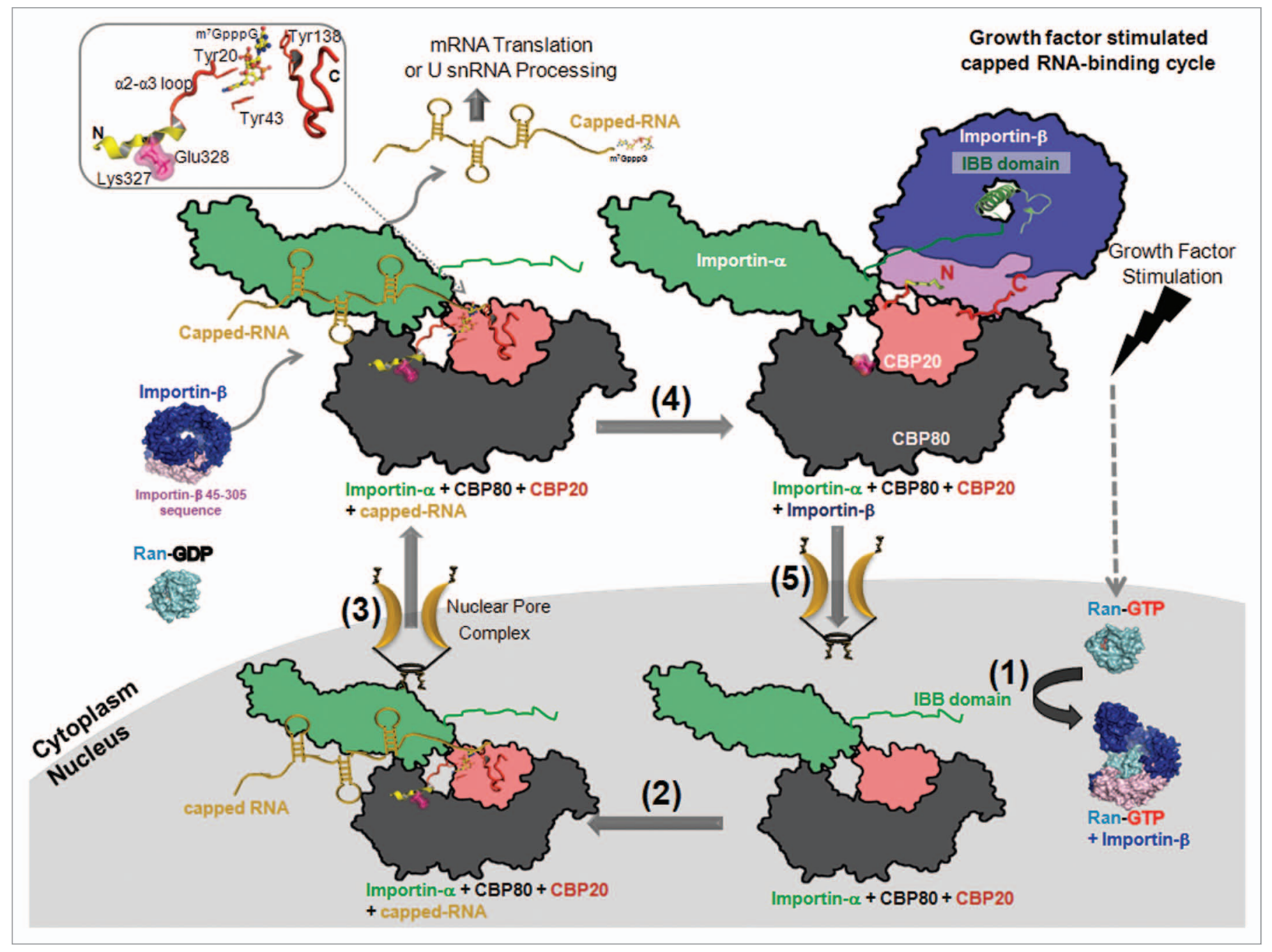

Figure 1. An overall view of how importins interact with the $C B C$ to regulate the cycle of capped-RNA binding in the nucleus and release into the cytoplasm.

Tyr43, in cap-binding has been inferred from previous structural determinations of the CBC, ${ }^{19,20}$ and have recently been shown to be involved in a two-step binding mechanism with the cap structure. ${ }^{14}$ Specifically, the binding mechanism involves a cooperation between Tyr20 and Tyr43, which engage in the recognition and stabilization of the 7-methylguanine moiety by cation- $\pi$ stacking, and then $\pi-\pi$ stacking between Tyr138 and the second nucleoside.

Finally, we asked whether one or both of the regions on CBP20, which are critical in cap-binding as described above, could also engage importin- $\beta$. Detailed mapping analysis combined with agarose native gel electrophoresis and RNA mobility shift experiments allowed us to determine that the $\mathrm{N}$-terminal 14 amino acid residues and the $\mathrm{C}$-terminal residues 126-156 of CBP20 are both critical contact points for importin- $\beta$ binding. ${ }^{22}$ The interaction between CBP20 and importin- $\beta$ could also be ablated by the addition of excess cap analog, suggesting that importin- $\beta$ competes with capped RNA for binding to CBP20. We additionally used small angle X-ray scattering (SAXS) experiments as an independent assessment of the supposition that importin- $\beta$, when bound together with importin- $\alpha$ to the CBC, should be in close contact to CBP20. Rigid body modeling of the known crystallographic structures for importin- $\beta$ (bound to the importin- $\beta$ binding domain from importin- $\alpha$ ) and the CBC-importin- $\alpha$ complex, to the SAXS data, juxtaposed CBP20 and importin- $\beta$. This model was further strengthened by the fact that the hydrodynamic parameters calculated for the proposed model agreed with the experimental values obtained by analytical ultracentrifugation. In total, these data allowed us to conclude that the importins have the ability to function as a switch to release capped RNA from the $\mathrm{CBC}$ by positioning importin- $\beta$ such that it is effectively able to out-compete the cap for binding to CBP20.

\section{Are the Importins Important in vivo?}

What about the in vivo relevance of this interaction? As stated above, it is attractive to envision the importins playing a 
critical function by facilitating the "passing of the baton", i.e., capped mRNA, from the CBC to eIF-4E in the cytosol in order for translation initiation to ensue. By triggering the release of RNA in the cytoplasm, the importins, upon nuclear re-entry, would also be facilitating the next round of capped-RNA binding by the CBC. Indeed, we wondered whether this importin-regulated, capped-RNA binding cycle of the $\mathrm{CBC}$ might underlie the growth factor-induced, capped-RNA binding event that we had reported on previously. ${ }^{8,9}$

Thus, to test the relevance of the importin interactions with the CBC in vivo, we asked if uncoupling the functional interaction between the $\mathrm{CBC}$ and the importins would disrupt the regulation of the $\mathrm{CBC}$ by growth factors. We designed a CBP80 NLS mutant that could no longer bind importin- $\alpha$ in a manner that we believed to be important for the proper positioning of importin- $\beta$ to induce the release of cap. To ensure proper localization, we introduced a functional NLS sequence onto the C-terminus of CBP80. While this CBP80 mutant was able to bind CBP20 and undergo nuclear localization, it was unable to bind capped RNA in response to heregulin, a growth factor which is particularly effective in stimulating the cap-binding capability of the CBC. These data demonstrated for the first time that the importins are essential regulators of CBC function in vivo by providing the molecular basis by which the $\mathrm{CBC}$ responds to growth factors. Additionally, they reaffirm the necessity of having importin- $\beta$ (residues 45-305) properly positioned as a result of the interaction between importin- $\alpha$ and CBP80, so that importin- $\beta$ can contact the $\mathrm{N}$ - and $\mathrm{C}$-termini of CBP20 to trigger the release of capped RNA (Fig. 1, step 4).

Our finding that importin- $\beta$ triggers cap-release by the CBC in cells has recently been corroborated by Sato and Maquat, ${ }^{23}$ who find that importin- $\beta$ prompts capped mRNA release in the cytoplasm following a CBC-dependent pioneer round of translation. The pioneer round of translation underlies the process of nonsensemediated mRNA decay, a quality control mechanism that ensures that mRNAs that would prematurely terminate translation are efficiently degraded. ${ }^{24}$ Following the pioneer round of translation, the $\mathrm{CBC}$ needs to be replaced by eIF-4E to allow the steady state translation initiation involved in protein synthesis. In their study, Sato and Maquat ${ }^{23}$ were interested in determining how this initial round of translation affects the conversion of the pioneer translation initiation complex into the steady state translation initiation complex. While the pioneer round of translation promoted the removal of exon junction complexes from spliced mRNA and facilitated the exchange of poly(A)binding protein $\mathrm{N} 1$ for poly $(\mathrm{A})$-binding protein $\mathrm{C} 1$ on the poly $(\mathrm{A})$ tail of the translated mRNA, it did not lead to the exchange of the CBC for eIF-4E at the $5^{\prime}$ cap of the mRNA. For the capped-mRNA to be handed off to the eIF-4E protein and enter steady states rounds of translation, the binding of importin- $\beta$ to the CBCimportin- $\alpha$-mRNA complex was found to be required.

\section{The Model}

The structural and biochemical profiles for the complexes formed between the $\mathrm{CBC}$ and the importins can now be discussed in the context of how the importins help to ensure the directional binding and release of capped RNA to/from the CBC in response to extracellular signals. An additional aspect of the model yet to be mentioned is the generation of Ran-GTP in response to growth factors. Indeed, the model for the importin-dependent regulation of the CBC capped-RNA binding cycle relies on nuclear Ran-GTP binding to importin- $\beta$ (depicted by step 1 in Fig. 1), thereby sequestering it from the CBC-importin- $\alpha$ complex in the nucleus. The effect of this step would be to impart high affinity cap-binding onto the CBC (step 2). It might therefore be predicted that growth factor signals leading to the accumulation of Ran-GTP would represent the penultimate signaling event in the activation of the cap-binding cycle of the CBC by the importins. We have recently shown this to be true in that Ran and the $\mathrm{CBC}$ respond in an identical fashion to extracellular signals and that the introduction of activated alleles of Ran into cells is sufficient to invoke a CBC cap-binding response (Ly et al., submitted). The CBC-
capped-RNA-importin- $\alpha$ complex is then exported into the cytosol (step 3), where Ran-GTP is hydrolyzed to Ran-GDP by the actions of RanGAP and RanBP1. ${ }^{25,26}$ This hydrolysis results in the dissociation of importin- $\beta$ from Ran so that importin- $\beta$ can engage the CBC-capped RNA-importin- $\alpha$ complex and stimulate the dissociation of the capped RNA (step 4). In the case of capped mRNA, this is a crucial step for the events leading to protein synthesis, since capped mRNA must first dissociate from the $\mathrm{CBC}$ in order to bind to eIF-4E for its translation into protein. For $\mathrm{U}$ snRNAs, the cap must undergo cytoplasmic hypermethylation as part of the process of formation of a mature U snRNP. ${ }^{27}$ The CBC-importin$\alpha$-importin- $\beta$ complex, depleted of capped RNA, is then imported into the nucleus (step 5). The growth factor signal activating Ran would re-start the cycle by causing the release of importin- $\beta$ from the CBC-importin- $\alpha$ complex and enabling the high affinity binding of another capped-RNA molecule. Thus, it is the differential effects on cap-binding affinity that occur when importin- $\alpha$ alone binds to the CBC, versus both importin- $\alpha$ and importin- $\beta$, as well as the ability of Ran to dictate whether or not importin- $\beta$ is part of this complex, that underlie the essential features of growth factor regulation, as well as direct capped-RNA release into the cytoplasm.

\section{Future Perspectives}

The Ran GTPase, through its interactions with the importins, plays an important role in nuclear cell biology by regulating nuclear transport, mitotic spindle assembly and nuclear membrane formation. Our work, ${ }^{22}$ and the subsequent study by Sato and Maquat, ${ }^{23}$ provide evidence for a new role for the importins and Ran in cells: directing capped RNA binding and release by the CBC. Not only do the importins and Ran mediate cap-binding, but they have the ability to do so in response to extracellular signals ${ }^{22}$ (Ly et al., submitted). What the consequence is for this level of regulation of the CBC by importins and Ran is an extremely interesting question, especially in light of growing evidence that Ran itself can play a direct 
role in cellular transformation (Ly et al., submitted). Thus, in the future we want to better understand how growth factors direct the activation of Ran. In particular, we will want to see whether this involves the growth factor-dependent regulation of RCC1 (i.e., the Ran-GEF) and what percentage of the total cellular pool of Ran is subject to growth factor regulation. We also will want to learn more about the transcripts that are being bound by the CBC in a growth factor-dependent manner and then delivered to the eIF-4E protein for subsequent translation. A further development would be to know more about the biological consequences of all of these processes, i.e., whether the growth factor regulation of $\mathrm{CBC}$ is also important in cellular differentiation (as well as cell growth), given that we earlier found that nerve growth factor is a strong activator of the CBC. ${ }^{8,9}$

\section{References}

1. von der Haar T, Gross JD, Wagner G, McCarthy JEG. The mRNA cap-binding protein eIF4E in posttranscriptional gene expression. Nat Struct Mol Biol 2004; 11:503-11.

2. Lewis JD, Izaurralde E. The role of the cap structure in RNA processing and nuclear export. Eur J Biochem 1997; 247:461-9.

3. Cheng H, Dufu K, Lee CS, Hsu JL, Dias A, Reed R. Human mRNA export machinery recruited to the $5^{\prime}$ end of mRNA. Cell 2006; 127:1389-400.

4. Ishigaki Y, Li X, Serin G, Maquat LE. Evidence for a pioneer round of mRNA translation: mRNAs subject to nonsense-mediated decay in mammalian cells are bound by CBP80 and CBP20. Cell 2001; 106:607-17.

5. Lejeune $\mathrm{F}$, Ishigaki $\mathrm{Y}, \mathrm{Li} \mathrm{X}$, Maquat LE. The exon junction complex is detected on CBP80-bound but not eIF4E-bound mRNA in mammalian cells: dynamics of mRNP remodeling. EMBO J 2002; 21:3536-45.
6. Maquat LE. Nonsense-mediated mRNA decay: splicing, translation and mRNP dynamics. Nat Rev Mol Cell Biol 2004; 5:89-99.

7. Mamane Y, Petroulakis E, Rong L, Yoshida K, Ler LW, Sonenberg N. eIF4E-from translation to transformation. Oncogene 2004; 23:3172-9.

8. Wilson KF, Fortes P, Singh US, Ohno M, Mattaj IW, Cerione RA. The nuclear cap-binding complex is a novel target of growth factor receptor-coupled signal transduction. J Biol Chem 1999; 274:4166-73.

9. Wilson KF, Wu WJ, Cerione RA. Cdc42 stimulates RNA splicing via the $S 6$ kinase and a novel $S 6$ kinase target, the nuclear cap-binding complex. J Biol Chem $2000 ; 275: 37307-10$.

10. Izaurralde E, McGuigan C, Mattaj IW. Nuclear localization of a cap-binding protein complex. Cold Spring Harb Symp Quant Biol 1995; 60:669-75.

11. Görlich D, Kraft R, Kostka S, Vogel F, Hartmann E, Laskey RA, et al. Importin provides a link between nuclear protein import and U snRNA export. Cell 1996; 87:21-32.

12. Li H, Tschudi C. Novel and essential subunits in the 300-kilodalton nuclear cap binding complex of Trypanosoma brucei. Mol Cell Biol 2005; 25:221626.

13. Oeffinger M, Wei KE, Rogers R, DeGrasse JA, Chait BT, Aitchison JD, et al. Comprehensive analysis of diverse ribonucleoprotein complexes. Nat Methods 2007; 4:951-6.

14. Worch R, Jankowska-Anyszka M, Niedzwiecka A, Stepinski J, Mazza C, Darzynkiewicz E, et al. Diverse role of three tyrosines in binding of the RNA 5 ' cap to the human nuclear cap binding complex. J Mol Biol 2009 385:618-27.

15. Niedzwiecka A, Marcotrigiano J, Stepinski J, Jankowska-Anyszka M, Wyslouch-Cieszynska A, Dadlez M, et al. Biophysical studies of eIF4E mcapbinding protein: recognition of mRNA 5 ' cap structure and synthetic fragments of eIF4G and 4E-BP1 proteins. J Mol Biol 2002; 319:615-35.

16. Niedzwiecka A, Darzynkiewicz E, Stolarski R. Thermodynamics of mRNA 5 ' cap binding by eukaryotic translation initiation factor eIF4E. Biochemistry 2004; 43:13305-17.

17. Duncan R, Milburn SC, Hershey JW. Regulated phosphorylation and low abundance of HeLa cell initiation factor eIF-4E suggest a role in translation control. Heat shock effects on eIF-4E. J Biol Chem $1987 ; 262: 380-8$
18. Izaurralde E, Lewis J, McGuigan C, Jankowska M, Darzynkiewicz E, Mattaj IW. A nuclear cap binding protein complex involved in pre-mRNA splicing. Cell 1994; 78:657-68.

19. Calero G, Wilson KF, Ly T, Rios-Steiner JL, Clardy JC, Cerione RA. Structural basis of $\mathrm{m}^{7} \mathrm{GpppG}$ binding to the nuclear cap-binding protein complex. Nat Struct Biol 2002; 9:912-7.

20. Mazza C, Segref A, Mattaj IW, Cusack S. Large-scale induced fit recognition of an $\mathrm{m}(7) \mathrm{GpppG}$ cap analogue by the human nuclear cap-binding complex. EMBO J 2002; 21:5548-57.

21. Krissinel E, Henrick K. Inference of macromolecular assemblies from crystalline state. J Mol Biol 2007; 372:774-97.

22. Dias SM, Wilson KF, Rojas KS, Ambrosio AL, Cerione RA. The molecular basis for the regulation of the cap-binding complex by the importins. Nat Struct Mol Biol 2009; 16:930-7.

23. Sato H, Maquat LE. Remodeling of the pioneer translation initiation complex involves translation and the karyopherin importin beta. Genes Dev 2009; 23:2537-50.

24. Ishigaki Y, Li X, Serin G, Maquat LE. Evidence for a pioneer round of mRNA translation: mRNAs subject to nonsense-mediated decay in mammalian cells are bound by CBP80 and CBP20. Cell 2001; 106:60717.

25. Coutavas E, Ren M, Oppenheim JD, D'Eustachio P, Rush MG. Characterization of proteins that interact with the cell cycle regulatory protein Ran/TC4. Nature 1993; 366:585-7.

26. Bischoff FR, Krebber H, Smirnova E, Dong W, Ponstingl H. Co-activation of RanGTPase and inhibition of GTP dissociation by Ran-GTP binding protein RanBP1. EMBO J 1995; 14:705-15.

27. Lührmann R, Kastner B, Bach M. Structure of spliceosomal snRNPs and their role in pre-mRNA splicing. Biochim Biophys Acta 1990; 1087:265-92. 